\title{
Organization of freelancers training to process economic information
}

\author{
V.D. Lobashev ${ }^{1 *}, A . A$. Talykh $^{2}$, and N.P. Shablikova ${ }^{3}$ \\ ${ }^{1}$ Petrozavodsk state University, Petrozavodsk, Russia \\ ${ }^{2}$ Petrozavodsk state University, Petrozavodsk, Russia \\ ${ }^{3}$ Petrozavodsk state University, Petrozavodsk, Russia
}

\begin{abstract}
Hiring freelancers significantly reduces the general costs: economic costs - payment for just specific work done, hours of work - the contractor is interested in the fastest execution of the order, consumer costs - the freelancer is self-sustainable, energy costs - the contractor is interested in energy saving. The stream of processed economic information is changing its quality. The exchange of structured data itself, being externally organized by the freelancers' efforts, significantly reduces operational (including transactional) costs.
\end{abstract}

\section{A problem statement}

In practical situations and collisions of an information society built on competition, a unique feature of the existence and activity of the information itself is revealed. It has acquired the qualities of a product participating in the creation of surplus value: the information acts simultaneously as object, means, instrument, goal and result of labor, acquiring and raising in the transitions of this process a unique value and a rigidly outlined market price, which develops, improves and continuously expands the area of its influence on the industrial relations quality transformation into systemically transformed informational and communicative ones [1].

New areas of application of ever more complex information technologies include varieties of software for the digital economy, distributed register technologies, analytical processing of arrays of ill-defined data, expert systems, and network technologies. The market steadily maintains and develops information increased demand areas presented by the market actors. The dynamic balance of the economic system at present is largely determined by the "information" economic resource. In the context of a specific production, the use of applied information saves highly restricted resources. In certain circumstances, information is adequate to the concept of a production factor, which, however, in its physical form, it is not [2].

Processing and further use (application) of the selected economic information is expedient up to the position of equality of the marginal utility of the studied unit of information to

${ }^{*}$ Corresponding author: ipcs-profped@yandex.ru 
the marginal costs of its acquisition. Objectively, information evolves, is ranked, generated and encoded as society develops, leaving behind fairly conservative social institutions. The value of an information resource, the latter being always subjective, is higher than the value of the carrier of the information under consideration [3].

\subsection{The objective of the work}

At the market, there are present and practically manifesting themselves antagonistic messages-claims of ownership areas for private special (including individually branded) information. In the general approach, the incompleteness of economic information presented in reality determines the financial and institutional system of the state, industry or company organizational and functioning foundations. It is these contradictions that freelancers are called upon to resolve in their practice. Freely functioning and freely oriented freelancers serve, stimulate and provide information movement between the levels of the available one, as well as one appearing in the course of additional search, thus, activating reference and information services of the market [4].

Freelancers, being a modern model of employment, in fact, reflect the quintessence of the most dynamic, flowing modern life. Taking the path of a separate organization of employment, a freelancer constructs a personal route of the life development vector - "DIY biography" (do-it-yourself biography). His career becomes patchy, sporadic, and acquires a project nature. Such specialists are most successful at virtual labor markets. Being extremely attractive, the acquired autonomy significantly increases the personal risks and, at the same time, the responsibility of the individual for independently made decisions [5].

A freelancer performs in the search for self-realization, but his/her job is closely related to the promotion of goods (specific tempered service), i.e. marketing incentive. In the market realities, these circumstances are more consistent with the intangible products exchange. This situation characterizes the post-industrial market, described as "cognitive capitalism" (A. Gorts 2010), exploiting primarily the employee's intellectual abilities and capabilities (skills). In fact, a freelancer, representing a "professionally organized" middle class, becomes an "information producer" (Castells 2000). The success of a freelancer's functioning critically depends on the coincidence, resonance of his/her qualifications and competence [6].

Freelancers institution, as a rule, does not receive income from the direct application of the information supplied by it. Thus, a freelance does not have an interest in information "delaying" and withholding. The leading goal remains a clearly expressed interest in spreading novelty in the form of selling their own services in the sphere of processing messages and then passing them on to a specific economic agent. In fact, a freelancer's "intended purpose" is to familiarize with and decipher the information accurately and qualitatively and to exclude the conditions and circumstances of asymmetric information flow. One of the main freelancer's tasks is to reduce the level of noise and distortion which can radically redefine management decisions. In this situation, the threshold for making effective management decisions ("cornerstone") is the limiting (sufficient and necessary) level and volume of reliable specific information [4].

Freelancers ultimately serve the highest (for a given customer, company, enterprise, association) level of management. By integrating messages from a group of freelancers who are not connected, the boss gets the opportunity to develop the company strategy. In their work freelancers immerse into the flow of messages being researched in the conditions of the constantly increasing uncertainty of the environment (announcements, requests, offers, etc.). In general case, this flow is random, which in practice forces freelancers to acquire very broad knowledge in various areas of application of their initiative [7]. The processed information, as a rule, requires the application of words fuzzy search technologies. Docu- 
ments indexing is done on the basis of a compiled language dictionary, permanently being supplemented by a dynamic dictionary of the entire volume of the collection under study. Ultimately, this makes it possible to achieve an adequate interpretation of the text content both by the customer (client), who ordered the analysis and by the contractor.

\section{Materials and the results of the research}

The problems of economic information analysts training (in practice - a refined orientation in the field of upcoming tasks) required the training methods and techniques development which would effectively improve the institutional mechanism of countering distortions of information, such as:

- traditions of working with information, internal instructions and restrictions;

- institutionalized separation of processed information levels;

- principles of the personnel structure formation;

- the amount of delegated authority in accordance with the level of the contractors qualifications; taking into account the mechanisms of price-setting and the risks occurrence of providing low-quality information by contractors;

- insurance procedures and mechanisms and degree of responsibility distribution among the contractors;

- the level of trust and stimulation of the quality of the tasks performed.

When organizing the freelancers training process, a formative experiment was applied, some attributes of which are the following:

- the main objective: to identify potential applicants for vacant positions of highly organized professional analysts, whose capabilities need to be identified and disclosed during the initial introductory course and subsequent testing;

- at this stage, primary training is performed (3-4 hours) with a demonstration of possible algorithms for processing information placed by service providers in various databases;

- in the future, a group of candidates (6-10 people) is formed, who continue to complete their studies for three days doing test assignments issued by the company leading managers; the total volume of texts is $15-17$ thousand characters;

- those who passed the control test receive work assignments for the analysis of economic information with the aim of its systematization, concentration and the necessary codification for further submission to the customer (this can be an individual entrepreneur, a company, or a project that is submitted for free access);

- processed information, as a rule, is allocated according to the subject: technology, movement of goods, services, etc;

- the algorithm for a freelancer's activity of this orientation consists of determining the intensity and demand depth of the selected topic and registering proposals which is, to varying degrees, are able to satisfy the indicated request; in fact, a refined brokerage task is being performed;

- in order to avoid collective collusion to slow down the execution of the task (it is paid by the piece), a deadline is indicated for each contractor, in addition, incentives are provided for the accelerated solution of the task;

- the criterion for completing tasks is the level of defects - it should not exceed $15 \%$ of the volume of the task (the number of solutions).

As the practice of such organizations (companies) shows, there is a very intensive change of contractors. Not more than $6-10 \%$ of those who started their activity at the beginning of the period (one year) work on a permanent basis. The number of errors made follow the $\chi 2$ law (the activity of three St. Petersburg firms has been examined for 5 years) with indicators $n=3-4$. 
Due to the noted specific features of the freelancers' activities involved in the economic information processing, when organizing their training, it is necessary to take into account the following circumstances and framework restrictions.

When examining the information, freelancers demonstrate the communicative-practical and cognitive motives (in accordance with the post regulations about the employment function and according to the task set by the short-term and long-term goals). Those motives permanently saturate the professional thesaurus, increasing and expanding the freelancers' qualification. An intensively formed thesaurus is aimed at penetrating into the essence of a phenomenon or a fact; it is characterized by a search orientation. The modern information society inclines its individuals to build personal, original thesauri, but in the future this seemingly fragmentary phenomenon obeys the interests of society, constructing a continuum of knowledge of the whole society, in particular subordinate and at the same time expressing the interests of each participant in industrial relations [8].

A freelancer's frame analysis provides an opportunity to identify the latent part of the ongoing interaction of all elements of the texts under consideration, as well as to assume the nature of the manifestation of procedures and functions that compose the model of activity participation of all carriers (authors) and consumers who receive information services [9].

Acquaintance with the text, its reading and conscious perception, interpreted as a process of strictly argued communication between the source of semantic information and the recipient, generates a counter movement of creating the image of the text and revealing its meaning. In the process of analytical-cognitive activity, the text is perceived and realized quite rhythmically, block-discretely, which is determined by the functional interaction of memory sections (short-term - interim - long-term), as well as the active manifestation of procedures-functions of perception motivation, attention, etc. In addition, the recurrent organization of the message content disclosure process is often used. The party receiving the text creates an individual model-tree of semantic saturation of each separately selected message in its own volume of the individual thesaurus and, as a rule, in this case, the communicative component fades into the background.

The argumentation of communication and the subsequent construction of a competent answer (decoding and recognition of the personal value) fully correlates with the perfection and degree of similarities of the perception structures and the response-answer variant formed by the task performer. It is revealing that, in a detailed analysis of the processes and motivation-oriented algorithms for creating and reading-perception of a text (educational, advertising, informational and broadcasting, etc.), there is a clear dependence of the final positive result of adequate perception of the sense and meaning of the text on the degree of similarities of the structures controlling the text generation and structures for constructing the respondent's thesaurus. It means that the tasks for freelancers must be selected in accordance with their "passport", which may be an applicant's mandatory testing result when being employed [9].

Considering the reading process as a form and an executive stage of mediated communication between a freelancer and the required information, it is necessary to distinguish three stages: planning - subordinate to the requirements of extracting linguistic information; performing - tracking the chain of actions and operations that form the field of the main motive and the goal of the message, the disclosure and understanding of which is performed in full accordance with the overall goal determined by the follow-up program based on the analysis of the perceived text. At the third stage, the structure of the argumentation of certifying the uniqueness of the concepts used in the interpretation of the text is analyzed separately.

Here, their own opinion-idea (of a freelancer) is formed about the meaning and significance of the text for the needs of the upcoming practical implementation of the disclosed 
(deciphered) novelty. The text transformation is accompanied by the development of a personal attitude towards the entire considered block (message). The statement concerning the value of the information is being planned. In some cases, the contractor is given the authority to make a decision on the final choice from a set of decision options in case of ambiguous situation assessment. "Unwittingly" he demonstrates management skills and, first of all, self-ruling in the course of a special event - transformation of the offered information and assessment of its consumer value. Actually, this is precisely his/her professional purpose.

The extracted information content essence is systematized at the levels of meaning and sense. In a qualitatively different plane, the following segments of information processing are distinguished: analysis of the text linguistic units; highlighting cause-and-effect relationships; penetration into the implicitly expressed meaning. When reading, semantic processing of the text occurs on the basis of technologies of qualitative logical-predicative semantic compression of information, which predetermines the transition from the superficial lexical and grammatical structure of the text to its internal content form, internal representation, the structure of semantic support points, penetration into the image of the text. Indicative is the permanent predicativization of information elements, proactively carried out by a freelancer to the degree and extent fully determined by perfect organization and the power of his/her thesaurus conceptual stock of [9].

In practice, the demonstrated critical thinking of the task performer to analyze the thematic content of the text is built in a tempered structure:

1. Formulation of goal-setting and frame-designating thesis;

2. A set of requirements and restrictions that clarify and specify the thesis;

3. Analysis of the available information blocks (descriptions, facts, principle approaches to the problem);

4. Development in real time mode and further on in the expanding field of concepts, formulating general provisions in the development, definitions, principles, with an outcome of consolidated and proved solutions.

Involuntarily occupying a "multi-factor" niche between the interests of acquaintance with the content essence, the goal-task requirements level, the volume of simultaneously evaluated information and the utilitarian usefulness of the analyzed information independently determined by himself/herself, a freelancer evaluates the text module by module: not rhythmically-prolonged, but forcefully-discretely-sequentially according to the logic of the outlined and allocated message block in conformity with the task algorithm.

The processing of unstructured text data is performed applying heuristic algorithms which take into account many third-party factors and counter their influence. As a consequence, under the influence of external factors, the algorithms themselves are intensively improved in the recursive mode. However, the information processing system should not be overly inert, otherwise its success is disturbed by inadequate to requests "auto-delays" generated as an attempt to show a violent reaction to incoming disturbances. The response should not be fussy. Resonant response frequencies often lead to the system breakdown through progressing chaos. Development of non-destructive positive feedback is required. Search for the border of the point of its decrease is relevant. At the forefront is the requirement of targeted information.

In some cases, the excessive originality of the message does not allow to recognize the essence of its novelty, and the vector of information elements is transformed into a set of semantic unit vectors, the uncertainty of the combinations of which represents the perceived message in the form of fan-shaped tuples, weakly connected by a common semantic constant. It should be recognized that the level of the message created as a result of the analysis and processing of the initial information should directly correlate with the abilities and capabilities of the prospective respondent, the latter is offered to perceive the information addressed to him/her, i.e. the comparability of the intelligences of the messages creator and 
consumer is the determining constant of the information (economic) activity ability. In this tuple, the freelancer occupies an intermediate position, he/she plays the role of an interpreter, relaxing the features of both initial information and restrictions on the application of the analysis result in time and in the area of implementation. In fact, he/she sets event framework boundaries for the application of his/her work result, which is used as an effective marketing tool.

Event marketing is a new technology that guides and, to a large extent, performs the product promotion process (information about a product, i.e. its brand) through the emotional sphere [10]. In this situation, the emotionally presented and expressed tool for the process success is focused on personal contacts between the consumer and the product, on the direct presentation of the product to the buyer through informal communication. Here, the market situation fully corresponds to the specific area of the activity-related manifestation of the regularities and requirements of event marketing. With an abundance of goods of approximately equal value, the attention of the target audience shifts from the rational to the emotional sphere. There is a clear increase in the manifestations of emotional intelligence, both of the client and of the consumer, which is contrastingly shown at the background of the forming information field [11].

Psychologists have proved that the disclosure of the meaning and sense of the semiotic system of information presentation is a necessary condition for holistic, systemic knowledge; an important factor of cultural understanding, operating with signs and verbal meanings. With a fairly selective access to the information meaning, the analyzed texts often exhibit inseparable processes of technological convergence and corporate mergers. The thought inherent in the message is always not fully expressed and it has to be supplemented and deciphered to the level of perceived information. The entropy of the message (the degree of uncertainty and the cost of finding a potential addressee) drops sharply over time.

The period of devaluation of the knowledge acquired as a result of message processing decreases exponentially. The observed paradox of perception and awareness-assimilation of the novelty is manifested in the lowering of the value of obtained and adopted knowledge over the information intensively received by the agent in the form of the updated thesaurus elements. The dynamics of the market is very tough in the competition, and critical analysis is replaced by the search for matches and analogues. In the conditions of the tough market competition, it becomes necessary to create certain reliability filters against the background of the manifestation of the "information fatigue" syndrome [12].

Filtering the unsystematic flow of information offered to a freelancer algorithmizes the requirements for the selection of data relevant to the initially set conditions - the customer's demands. To solve this problem on can use keywords sets, educational aids, sets of training tables of the most frequent and serious errors, abbreviations tables, etc.

In the processes of familiarization with the newly arriving (according to the production tasks) information, all perceived message alphabets signs (language constants, sounds, symbols, emotions) are continuously compared with the standards that previously had "filled" the thesaurus. Subsequently, on a competitive basis, they are replaced by the newly acquired ones and then approved as priority elements of the updated content. This is the essence of training carried out according to the principle of the novelty priority and the truth of the incoming and evaluated information. It can be carried out as an element of selfeducation. But training is much more productive when considering and analyzing an outside opinion, presented in the form of some alternative, attracted and competitively participating in the processes of a freelancer's thesaurus elements forming.

In a pedagogical situation of this type, there can be both an atmosphere of revolutionary renewal and an expansive interpretation of trustworthy evidence of a thesaurus separate element status significance addition. 
The next control stage is filled with the procedures of comparison, contrasting one's own understanding with the possible opinion of the inspector. In process with the acquisition of professional skills, the reasoned self-control of the text modal information the comes into play. The critical correlation of the text with the student's experience, recorded in the forms-images of the standards, carried out in the ongoing analysis, establishes the links between the levels "text - reality" and "text - recipient", expressed and consolidated in the presented opinions and defended arguments [9].

The main requirements for optimizing information assessment processes are that the most rational information provided for freelancers for subsequent processing and analysis must be allocated by focus, specialized according to the conditions and requirements of the solutions being developed, etc. The very specific professionalization of this class of employees becomes an indispensable condition.

One of the defining moments in the processing of incoming information is its categorization. Further on, the processes of setting parameters and criteria for the reliability and value of information (practically intelligent filters), as well as the mental processes of creation, control, management, categorization, etc. reflect the mental activity of the individual. Each aspect of thought is characterized by the presence of specific elements and mechanisms. Analysis of the literature allows us to single out six aspects of the thought functioning: sensory-perceptual, motoric, emotional, programming, commanding and conceptual [8].

In the course of practical activity, a freelancer acquires a complex of abilities to use the continuously accumulated array of information, while at the same time gaining specific training in transferring knowledge from one situation to another. The verbal intelligence is significantly improving, which is considered as an increasing ability for the extended application of general cognitive schemes and, on the basis of mixing special knowledge and algorithms of basic procedures - for extracting information from the formed thesaurus. Here, abilities are being developed and affirmed, readiness is being perfected, competencies for organizing reasoning procedures and verbal material deep understanding in the situations of seeking analytical problems solutions are being formed [13].

A freelancer' information competence as the degree of the integration of his/her abilities and willingness to use when operating with information in the professional field is realized in professional activity in the form of reflexive-communicative, professional-activity and value-motivational functions. The education (level of training) of such a specialist should ensure his readiness to show in practice the properties of subjectivity, dynamism, polyfunctionality, accumulation, sociability, collaboration, and to present in his/her decisions a technological singularity, electronic institutionalism, autopoiesis, etc.

Formed in the process of intensive practical activity, a freelancer's thesaurus acquires the features and properties of functional consistency. During the development of information retrieval and professional thesauri, the personality structure is the object of active modernization. The basic element in this situation is not an innovative concept, but a concept which is a fusion of meaning and sensory perception expressed in the analyzed signs (professional alphabet), an internal image extracted from long-term memory, from the depths of a previously formed thesaurus area. This element is connected with other concepts not only by logical, but also by value relationships.

One of the most effective methodological techniques for organizing an intensive search for an optimal solution to an information problem is collective search. The construction of a collective search can be represented by a multi-agent system using metasearch technologies, which successfully ensures the effective formation of address-theme oriented complex collections of achieved solutions. The projected and created collective subject is transformed into some single integral formation, a collective mind [14]. The solution level rises crucially. However, a personal and collective thesaurus requires, for its consolidation, the 
conduct of discursive practices, in which are vividly, reliably and professionally realized the receptive skills, obtained as a result of collective creativity and applied to extract the meaningful essence of a convoluted and thematically encoded text.

\section{Conclusions}

The freelancers' training and instructing carried out during the hiring process has an episodic nature, and the method of organizing it requires careful and detailed development. The following provisions can be noted as initial conclusions:

- at the final stages of training as test means the "survey" matrices of the thesaurus level on the topic allocated to the group of freelancers can be used;

- the dialogue of two individuals (task performers) with different terminologically oriented thesauri is of interest and worth considering. That means that in pair work of professionals of various special training during the exchange of options for solving a given problem the "effect of identifying an element of complementarity" may appear [15];

- the freelancer's thesaurus formed during the course of professional improvement training must be stored with vocabulary conceptual entries, i.e. expanded interpretations of concepts, revealing the broadest field of interpretation of the concept itself during primary education [16];

- as a result, it is especially important to define the applicant's personal volume and orientation of the thesaurus;

- in multi-agent systems, the agent's behavior, the choice of the optimal strategy in particular, depends on the other agents' strategy of; multi-agent systems with pronounced decentralization at the same time, as a rule, are characterized by a significant correlation of behavior elements (decision-making) both among individual task performers and subordination to the central task [17];

- orientation of the educational process to the implementation of a functionally cumulative complementary complex of the system of elements of culture, text, language and thinking allows to solve practice-oriented educational tasks of professionals training [18];

- cluster analysis of the control tests results is very productive in differentiating agents;

- one must understand that the creation of a new thesaurus element is predetermined by past experience, at least by its initial presence. Experience in this situation "determines", anticipates the direction of development of the training freelancers theory [19];

- the integration of rational and intuitive experience, the use of various forms and types of information presentation create new opportunities for the interpretation and creation of an a new fact or concept inner image [20].

These provisions allow us to speak about the role of subjective experience as a decisive factor in training aimed at developing a freelancer's personality seeking for improvement.

\section{References}

1. V.I. Grachev, The information marketing properties in the modern socio-cultural communication system, Izvestiya: Herzen university journal of humanities \& sciences, 71, 207-214 (2008)

2. T.W. Malone, R. Laubacher, M.S. Scott Morton, The Dawn of the E-Lance Economy, Inventing the organizations of the 21st century, 103-114 (London, 2003)

3. G. Nies, R. Pedersini, Freelance Journalists in the European Media Industry (2003) 
4. A.V. Grigoriev, The assessment of uncertainty based on the maximal entropy principle in the process of economic decision making, Vestnik of Krasnoyarsk State Agrarian University, 11, 24-29 (2006)

5. T. Schultz, Investment in Human Capital, Economic Growth - an American Problem. Englewood Cliffs, 35 (1964)

6. M. Benz, B. Frey, The Value of Doing What You Like: Evidence From the SelfEmployed in 23 Countries, Journal of Economic Behavior and Organization, 68 (3-4), 445-455 (2008)

7. Inventing the organizations of the 21st century, T.W. Malone, R. Laubacher, M.S. Scott Morton (London, 2003)

8. V.Y. Baganov, The main aspects of decision making in the conditions of uncertainty, Azimuth of scientific research: economics and administration, 2 (27), 54-68 (2019)

9. I.V. Slesarenko, The peculiarities of the statement formation process based on the argumentation elements methods hierarchical structure, Vestnik of Tomsk State University, 291, 260-264 (2006)

10. N. Bonds, N.C. Dragonetti, K. Jaconsen, G. Roos, The knowledge toolbox: a review of the tools available to measure and manage intangible resources, Europian Management Journal, 17 (4), 391-402 (1999)

11. A.A. Manikhin, Event-marketing position in the marketing communication complex, Creative economy, 4, 135-143 (2010)

12. N.R. Shevko, Actual problems of information security provision in the contemporary society, Vestnik of Kazan Law Institute of MIA of Russia, 2 (8), 57-59 (2012)

13. Zh.V. Korneva, E.V. Ioda, N.N. Krivykh, The conditions of information economy development, Vestnik of Tambov University. Series: Humanities, 11, 20-24 (2009)

14. A.A. Talykh, V.D. Lobashev, The peculiarities of education process construction in the Technology Education sphere, Vestnik of Kostroma State University named after N.A. Nekrasov, 22 (1), 201-205 (Kostroma, 2016)

15. W. Saabeel, Model of Virtual Organization Structure \& Process, Electronic Journal of Organizational Virtualness, 4 (1) (2004)

16. L.G. Medvedeva, The principles of professionally-oriented thesaurus (based on the teaching experience in TSU Law Institute), Vestnik of Tomsk State University, 297, 47-49 (2007)

17. C. Welzel, The Theory of Human Development: A Cross-Cultural Analysis, European Journal of Political Research, 42, 341-379 (2003)

18. V.D. Lobashev, I.V. Lobashev, Motivation in the processes of professional education, 543 (LAP LAMBERT Academic Pudlishing, 2012)

A. Price, Human Resource Management in a Business Context, Cengage Learning EMEA (2007)

19. H.R. Bowen, Investment in Learning, San Francisco etc: Jossey-Bass, 362 (1978) 